\title{
3D dynamic pituitary MR imaging with CAIPIRINHA: initial experience and comparison with 2D dynamic MR imaging.
}

\section{$\operatorname{AUTHOR}(S)$ :}

Fushimi, Yasutaka; Okada, Tomohisa; Kanagaki, Mitsunori; Yamamoto, Akira; Kanda, Yumiko; Sakamoto, Ryo; Hojo, Masato; Takahashi, Jun C; Miyamoto, Susumu; Togashi, Kaori

\section{CITATION:}

Fushimi, Yasutaka ...[et al]. 3D dynamic pituitary MR imaging with CAIPIRINHA: initial experience and comparison with 2D dynamic MR imaging.. European journal of radiology 2014, 83(10): 1900-1906

\section{ISSUE DATE:}

2014-10

\section{URL:}

http://hdl.handle.net/2433/197929

\section{RIGHT:}

(c) 2014 Elsevier. Licensed under the Creative Commons Attribution-NonCommercial-No Derivatives 4.0 International http://creativecommons.org/licenses/by-nc-nd/4.0/. NOTICE: this is the author's version of a work that was accepted for publication in European journal of radiology. Changes resulting from the publishing process, such as peer review, editing, corrections, structural formatting, and other quality control mechanisms may not be reflected in this

document. Changes may have been made to this work since it was submitted for publication. A definitive version was subsequently published in European journal of radiology, 83(10), doi:10.1016/j.ejrad.2014.06.018; 許諾条件により、本 文ファイルは2015-07-02に公開.; This is not the published version. Please cite only the published version.; この論文は出 版社版でありません。引用の際には出版社版をご確認ご利用ください。 
Title

3D dynamic pituitary MR imaging with CAIPIRINHA: initial experience and comparison with 2D dynamic MR imaging 
Methods: Participants comprised 32 patients who underwent dynamic pituitary MR imaging due to pituitary or parasellar lesions. 3D dynamic MR with CAIPIRINHA was performed at $3 \mathrm{~T}$ with 20 -s-interval, precontrast, 1 st to 5 th dynamic images. Normalized values and enhanced ratios (dynamic postcontrast image values divided by precontrast ones) were compared between 3D and 2D dynamic MR imaging for patients with visual identification of posterior lobe and stalk.

Results: In 3D, stalk was identified in 29 patients and unidentified in 3, and posterior lobe was identified in 28 and unidentified in 4. In 2D, stalk was identified in 26 patients and unidentified in 6 patients, and posterior lobe was identified in 15 and unidentified in 17. Normalized values of pituitary posterior lobe and stalk were higher in $3 \mathrm{D}$ than $2 \mathrm{D}$ $(\mathrm{P}<0.001)$. No significant difference in enhancement ratio was seen between $3 \mathrm{D}$ and 2D.

Conclusions: 3D dynamic pituitary MR provided better identification and higher normalized values of pituitary posterior lobe and stalk than 2D. 


\section{TEXT}

\section{INTRODUCTION}

Dynamic contrast-enhanced MR imaging of pituitary adenomas has been

widely used in clinical practice ${ }^{1-3}$. The normal pituitary typically enhances within the

first 1-2 min after contrast injection and shows an enhancement pattern consistent with the vascular architecture of the pituitary, with the posterior lobe enhancing earlier than the anterior lobe. Dynamic MR imaging has provided beneficial information for various situations by demarcating normal pituitary gland: microadenomas ${ }^{1,4}$, Cushing disease ${ }^{5,}$ ${ }^{6}$, ectopic pituitary gland ${ }^{7}$, idiopathic growth hormone deficiency ${ }^{8}$, Tolosa-Hunt syndrome ${ }^{9}$, and adjacent tissues ${ }^{10}$. Asymmetrical pituitary enhancement on dynamic MR imaging is also observed due to localized venous congestion caused by cavernous sinus arteriovenous fistula ${ }^{11}$.

Dynamic pituitary MR imaging has usually been performed in coronal planes rather than sagittal planes ${ }^{1}$. Small lesions between slices, or lesions located at the far anterior or far posterior aspect of the pituitary glands, might be overlooked on MR imaging using only coronal planes. The use of consecutive coronal and sagittal dynamic images to acquire superior diagnostic accuracy rate for pituitary microadenomas in comparison to imaging using only coronal planes has been reported ${ }^{12}$, but required a 
double or triple volume of contrast medium for the second injection to overcome the

contrast induced by the first injection of contrast medium.

Sagittal dynamic MR enables evaluation of the posterior lobe, which

demonstrates a lack of early enhancement in lymphocytic hypophysitis probably due to secondary inflammatory changes in some pediatric patients with central diabetes

insipidus ${ }^{13}$. Central diabetes insipidus has been associated with embolization of the meningohypophyseal trunk of the internal carotid artery ${ }^{14}$, systemic fat embolism and transient ischemic attack ${ }^{15}$, suggesting the importance of recognizing posterior lobe enhancement.

Pre- and post-contrast 3D gradient-echo imaging techniques provide better spatial resolution of pituitary lesions and adjacent structures with thin slices that can be reformatted in orthogonal directions for the detection of pituitary microadenomas ${ }^{16}$. Accurate volumetric changes in pituitary adenoma have been assessed using longitudinal MR image registration of 3D images ${ }^{17}$. However, 3D dynamic imaging suffers from a tradeoff between temporal and spatial resolutions.

Phase and partition encoding directions can be applied for parallel encoding in 3D imaging by utilizing sensitivity variations in both encoding directions of multiple arrays of the head coil. The controlled aliasing in parallel imaging results in higher 
acceleration (CAIPIRINHA) method has recently been introduced ${ }^{18}$, and modifies the appearance of aliasing artifacts in parallel imaging using these modified phase encoding patterns under generalized autocalibrating partially parallel acquisitions (GRAPPA) ${ }^{19}$. Reordering of phase and partition encoding is conducted in CAIPIRINHA by shifting sampling positions from the normal positions in undersampling, which leads to shift aliasing so that sensitivity variations based on the underlying receiver array coil can be exploited efficiently ${ }^{18}$. The CAIPIRINHA method results in further improvement in parallel imaging reconstruction conditions and decreases to both noise and artifacts.

In this study, a 3D volumetric interpolated breath-hold examination (VIBE) sequence with CAIPIRINHA (3D dynamic MR) was applied for high temporal-resolution 3D dynamic pituitary studies. To evaluate the validity of 3D dynamic MR, (i) a phantom study for 3D dynamic MR was conducted along with (ii) a comparison study between 3D dynamic MR and conventional spin echo 2D dynamic MR, with special emphasis on demarcation of the pituitary posterior lobe and stalk.

\section{MATERIALS AND METHODS}

\section{Phantom study}

MR imaging for a 2\% agar phantom was performed using a 3-T MR unit 
(Magnetom Skyra; Siemens, Erlangen, Germany) with 32-channel head coil. Thirty measurements of 3D VIBE with CAIPIRINHA were conducted. For constructing the sample pattern of CAIPIRINHA in this study, a 3D GRAPPA pattern with acceleration of "phase encoding direction $=2$ ", and "partition encoding direction $=2$ " was adopted as the sampling pattern for CAIPIRINHA (net reduction factor of 4). The partition encoding direction was sheared by "Delta Shift PAR" per line in partition, as described in previous reports ${ }^{18}$ : "Delta Shift PAR $=1$ ", where every second phase encoding table in the phase encoding direction is shifted by 1 in the partition encoding direction ${ }^{18}$. Thirty measurements of 3D VIBE without parallel imaging were also obtained for calculation of the geometry factor (G-factor). The 3D VIBE sequences were performed with coronal acquisition (TR, $5.5 \mathrm{~ms}$; TE, $1.83 \mathrm{~ms}$; flip angle, $7^{\circ}$; matrix, $192 \times 192$; field of view (FOV), $183 \times 183 \mathrm{~mm}$; isotropic voxel of $1 \mathrm{~mm}$; 52 slices; acquisition time, 20s).

Signal-to-noise ratio (SNR) was calculated in a pixel-wise manner from mean signal intensity divided by standard deviation through 30 measurements ${ }^{20,21}$. G-factor was calculated from SNRs with and without parallel imaging (Fig. 1).

\section{Patients}


This retrospective study was approved by the institutional review board. The 32

consecutive patients enrolled in this study (14 males, 18 females; mean age, $52.8 \pm 17.2$

years) and they had undergone dynamic MR imaging due to pituitary or parasellar lesions between June 2012 and December 2012 and the final diagnosis were as follows: pituitary macroadenoma (preoperative, $n=6$; postoperative, $n=13)$, microadenoma $(n=3)$, Rathke's cleft cyst $(n=2)$, meningioma $(n=4)$, or no abnormal findings $(n=4)$. Patients with craniopharyngioma and inflammatory disease such as lymphocytic hypophysitis were excluded due to the history of diabetes insipidus ${ }^{22}$.

Two-dimensional (2D) dynamic MR had previously been performed for 22 of the 32 patients who underwent 3D dynamic MR at our institute, since most have been undergoing annual MR scans to check residual tumor size or disease condition. Matched for pituitary disorders with the remaining 10 patients who had undergone 3D dynamic MR only, an additional 10 patients were randomly selected from the hospital reporting system, and 32 patients were thus also enrolled for 2D imaging (10 males, 22 females; mean age, $52.7 \pm 16.7$ years).

\section{MR imaging parameters}

\section{D dynamic MR}


All 3D dynamic MR was performed using a 3-T MR unit (Magnetom Skyra,

Siemens, Erlangen, Germany). Dynamic studies were performed with 3D

VIBE-CAIPIRINHA with coronal acquisition (TR, $5.5 \mathrm{~ms}$; TE, $1.83 \mathrm{~ms}$; flip angle, $7^{\circ}$; matrix, $256 \times 256$; FOV , $180 \times 180 \mathrm{~mm}$; isotropic voxel, $1 \mathrm{~mm}$; 52 slices; acquisition time, $20 \mathrm{~s}$ ). A slab thickness of $52 \mathrm{~mm}$ was adopted so that sufficient pituitary and parasellar areas must be covered to obtain better sagittal and axial multiplanar reconstruction (MPR) views. The acceleration factor for the in phase encoding direction was 2, the in partition encoding direction was 2, and "Delta Shift PAR" per line in partition encoding was 1 , corresponding to the pattern described in the phantom study. Dynamic imaging started with the first precontrast image, followed by a second image $10 \mathrm{~s}$ after rapid injection $(4 \mathrm{ml} / \mathrm{s})$ of $0.1 \mathrm{mmol} / \mathrm{kg}$ of gadolinium-based MR contrast agent, with four subsequent serial images obtained over $90 \mathrm{~s}$ at 20 -s intervals (1st to 5 th scans: $10,30,50,70$, and $90 \mathrm{~s}$, respectively).

\section{D dynamic MR}

All 2D dynamic MR was performed using 3-T MR units (Magnetom Skyra or Magnetom Trio; Siemens, Erlangen, Germany) or 1.5-T MR units (Magnetom Symphony, Siemens, Erlangen, Germany) prior to 3D dynamic scans (846 \pm 645 days). 
The 2D dynamic images were acquired in the coronal or sagittal plane with FSE

sequences (TR, $500 \mathrm{~ms}$; TE, $12 \mathrm{~ms}$; matrix, 256×192; echo train length, $8 \mathrm{~s}$; FOV, $180 \times$ $180 \mathrm{~mm}$; and acquisition time, $13 \mathrm{~s}$ ). Four contiguous 3-mm-thick sections were imaged simultaneously, so that both pituitary stalk and the dorsal region of the sella turcica were imaged. Dynamic imaging started with the first precontrast image, followed by a second image $15 \mathrm{~s}$ after rapid injection $(4 \mathrm{ml} / \mathrm{s})$ of $0.05 \mathrm{mmol} / \mathrm{kg}$ of gadolinium-based MR contrast agent, with four subsequent serial images obtained over $75 \mathrm{~s}$ at $15-\mathrm{s}$ intervals $(15,30,45,60$, and $75 \mathrm{~s})$.

\section{Postimaging analysis}

Data from 3D and 2D dynamic MR were uploaded to the DICOM viewer using an Aquarius iNtuition Server (TeraRecon; Foster City, CA, USA). Zero-filling interpolation was automatically applied for $2 \mathrm{D}$ data in the slice direction on Aquarius iNtuition Server. Two neuroradiologists (_._., 15 years of experience; _._., 17 years of experience) assessed visualization and enhancement of the posterior lobe and stalks. The posterior lobe and stalk were graded as "identified" when visualization and enhancement of these structures were identified in precontrast and dynamic series. Otherwise, these structures were graded as "unidentified". Consensus reading was 
adopted. Region of interest (ROI) analysis of "identified" posterior lobes and stalks was performed on ImageJ software (http://rsbweb.nih.gov/ij/index.html) using sagittally reconstructed images with referring images of Aquarius iNtuition Server. ROI comprising air in the sphenoid sinus just below the pituitary lobe was calculated so that values could be used to normalize the intensity of the posterior lobe and stalk among patients for all dynamic enhanced phases as follows: a) values and coefficients of variance $(\mathrm{CV})$ of air intensity were calculated for precontrast (Pre) and postcontrast dynamic images; b) normalized values for the pituitary lobe and stalk on Pre and postcontrast dynamic images were analyzed between $3 \mathrm{D}$ and $2 \mathrm{D}$; and c) values without normalization of postcontrast dynamic images divided by values from the precontrast image were analyzed as the enhancement ratio between 3D and 2D.

The posterior lobe on MR images was defined as follows: a thin structure located in the sella turcica that shows hyperintensity or intermediate intensity with arterial enhancement in the patients without any history of diabetes insipidus. The anterior lobe was excluded from analysis, since a normal anterior lobe was heavily displaced in patients with pre- and postoperative macroscopic adenoma in this study.

\section{Statistical analysis}


was used for statistical analysis. Repeated-measures analysis of variance was performed for the air intensity comparisons of Pre and postcontrast dynamic images between 3D and 2D. Welch's t test was used for normalized values for the pituitary lobe and stalk, and for comparison of values from postcontrast dynamic images divided by precontrast image values between $3 \mathrm{D}$ and $2 \mathrm{D}$ dynamic images. $\mathrm{P}<0.05$ was set as the level of statistical significance.

\section{RESULTS}

\section{Phantom study}

G-factor calculated for 3D VIBE with CAIPIRINHA is presented in Figure 1. With CAIPIRINHA, G-factor is reduced at the central part of the image and slab, and increased at the peripheral part of the image and slab (Fig. 1).

\section{Patient study}

The success rate of the dynamic pituitary study was $100 \%$.

On 3D dynamic imaging, the stalk was identified in 29 patients and unidentified in 3 , while the posterior lobe was identified in 28 and unidentified in 4 . On 
2D imaging, the stalk was identified in 26 patients and unidentified in 6 , while the posterior lobe was identified in 15 and unidentified in 17. Representative images are shown in Figures 2-4.

\section{Comparison between 3D and 2D dynamic images}

The results for air intensity in the sphenoid sinus just below the pituitary lobe are shown in Table 1. Significant differences were seen in pre- and postcontrast dynamic images (2nd, 3rd, 4th and 5th) $(\mathrm{P}=0.0216,0.009,0.0014$ and 0.0017 , respectively) between 3D and 2D dynamic images.

Patients with positive identification of both the posterior lobe and stalk were included for comparison, comprising 28 patients from 3D imaging and 15 patients from 2D imaging. Normalized values of precontrast and dynamic contrast images were compared at Pre, 1st, 2nd, 3rd, 4th and 5th between 3D and 2D dynamic images. Mean and standard error are shown in Figure 5. Normalized values of the posterior lobe and stalk were higher in 3D than in 2D. Significant differences were seen for all comparisons $(\mathrm{P}<0.001$ each $)$.

Enhancement ratio was derived using non-normalized values from postcontrast dynamic images divided by precontrast image values. Enhancement ratio of the 
posterior lobe and stalk were compared using 1st/Pre, 2nd/Pre, 3rd/Pre, 4th/Pre and 5th/Pre between 3D and 2D dynamic images. No significant differences were seen for any comparisons. Mean and standard error are shown on Figure 6.

\section{DISCUSSION}

The use of 3D dynamic pituitary MR with CAIPIRINHA clearly showed 3D dynamic contrast changes in the pituitary gland. Parallel imaging was applied for both phase encoding and partition encoding directions, successfully shortening the scan time and improving spatial resolution. Coil sensitivity variations can be exploited more efficiently in multiple dimensions using CAIPIRINHA, resulting in a more robust parallel imaging reconstruction. The in-plane distribution of G-factor values was relatively inhomogeneous, but higher values were found at the periphery. This might also be good for the evaluation of pituitary lesions, since G-factor seemed low in central parts of images in the imaging slab.

Normalized values of the pituitary posterior lobe and stalk were higher at 3D than at $2 \mathrm{D}$, but no significant differences in enhancement ratio were seen between $3 \mathrm{D}$ and 2D. Few comparisons of the rate of positive identification of the posterior pituitary lobe on 3D and 2D dynamic studies appear to have been reported. In 3D dynamic 
pituitary MR imaging, hyperintensity of the posterior pituitary lobe on pre-contrast images and enhancement of the posterior lobe were more easily recognized than in $2 \mathrm{D}$ dynamic pituitary MR imaging because of the better spatial resolution. The $2 \mathrm{D}$ dynamic study was acquired with a 3-mm slice thickness in the coronal plane, which by its nature was unsuited for evaluation of the posterior lobe. In addition, it was often difficult for 2D dynamic MR imaging to cover all the pituitary lesions in cases of macroadenoma. Despite better spatial and temporal resolution, fewer differences in enhanced ratio of the posterior lobe and stalk were seen between 3D and 2D dynamic MR imaging.

Hyperintensity of the pituitary posterior lobe was not identified for 4 cases in this study. Failure to identify the posterior pituitary lobe might occur for patients without diabetes insipidus ${ }^{23}$. Postoperative posterior hyperintensity is sometimes difficult to identify due to hematoma or previous surgical procedures ${ }^{24}$. Patients with a history of surgery, irradiation, or treatment with medications such as dopamine receptor agonists were included in the present study, and the posterior pituitary lobes were invisible even on routine 3D sagittal $\mathrm{T} 1$ sequences for all patients with negative identification on 3D dynamic MR imaging.

Superior hypophyseal arteries, inferior hypophyseal arteries and trabecular artery are known to supply arterial blood to the pituitary gland, entering the pars 
nervosa or posterior gland. Some of the arterial branches form a capillary mesh in the region of the median eminence, with venules extending into the pars tuberalis and then the anterior lobe and forming the hypophyseal portal system. Dynamic studies provide temporal and spatial dynamic information on the complicated vascular supply of the pituitary gland. Dynamic MR studies also visualize response to stimulation with hypothalamic releasing hormones, showing increased enhancement of the pituitary gland ${ }^{25}$. Use of 3D dynamic CT of the pituitary is reportedly superior to MR imaging for assessing lateral tumor margins and the sellar floor at the sphenoid sinus ${ }^{26}$, but multiple irradiation exposures remain controversial. The previous study performed alternative acquisitions in several planes to obtain sagittal and coronal information on the pituitary gland ${ }^{27}$, whereas $3 \mathrm{D}$ dynamic pituitary MR with CAIPIRINHA in this study was suitably performed with acceptable temporal and spatial resolution.

Several limitations in this study must be considered. The duration of dynamic scanning differs between 3D and 2D dynamic studies. Use of the same imaging time as 2D dynamic MR results in a narrower range of coverage for 3D dynamic MR. We chose wider coverage for 3D dynamic MR so that better MPR images could be obtained. Second, the ROI of air in the sphenoid sinus just below the pituitary lobe was adopted for normalization. ROI of the pons has been adopted for comparison in previous studies 
because of signal intensity invariance ${ }^{7,28}$, but both 3D and 2D dynamic images failed to cover the pons. Furthermore, tissue adjacent to the pituitary should be referred to in the context of parallel imaging due to the complicated sampling reduction, with a net reduction factor of 4, applied in the CAIPIRINHA method in this study. The G-factor of the phantom revealed the great differences between central and peripheral parts of the image and the partition, so comparisons should be performed with the structure located in the central part of the image and partition. Two-region approach should not be used for SNR calculation for parallel imaging ${ }^{20}$, and the signal of the pituitary was compared with air just below the pituitary lobe although the $\mathrm{CV}$ of air intensity was relatively large on 3D imaging. Third, identification of microadenoma was not performed in this study. More clinical research is needed to evaluate the efficiency of identifying microadenoma using 3D dynamic MR.

In conclusion, 3D dynamic pituitary MR provided better identification of posterior lobe and stalk than $2 \mathrm{D}$, normalized values for pituitary posterior lobe and stalk were higher on $3 \mathrm{D}$ than on $2 \mathrm{D}$, and no significant difference was seen concerning enhancement ratio between 3D and 2D. 


\section{REFERENCES}

[1] Miki Y, Matsuo M, Nishizawa S, et al. Pituitary adenomas and normal pituitary tissue: enhancement patterns on gadopentetate-enhanced MR imaging. Radiology 1990;177(1):35-8.

[2] Sakamoto Y, Takahashi M, Korogi Y, Bussaka H, Ushio Y. Normal and abnormal pituitary glands: gadopentetate dimeglumine-enhanced MR imaging. Radiology 1991;178(2):441-5.

[3] Miki Y, Asato R, Okumura R, Hua F, Konishi J. Contrast enhanced area of posterior pituitary gland in early dynamic MRI exceeds hyperintense area on T1-weighted images. J Comput Assist Tomogr 1992;16(6):845-8.

[4] Bartynski WS, Lin L. Dynamic and conventional spin-echo MR of pituitary microlesions. AJNR Am J Neuroradiol 1997;18(5):965-72.

[5] Friedman TC, Zuckerbraun E, Lee ML, Kabil MS, Shahinian H. Dynamic pituitary MRI has high sensitivity and specificity for the diagnosis of mild Cushing's syndrome and should be part of the initial workup. Horm Metab Res 2007;39(6):451-6.

[6] Portocarrero-Ortiz L, Bonifacio-Delgadillo D, Sotomayor-Gonzalez A, Garcia-Marquez A, Lopez-Serna R. A modified protocol using half-dose gadolinium in dynamic 3-Tesla magnetic resonance imaging for detection of ACTH-secreting pituitary tumors. Pituitary 2010;13(3):230-5.

[7] Takahashi T, Miki Y, Takahashi JA, et al. Ectopic posterior pituitary high signal in preoperative and postoperative macroadenomas: dynamic MR imaging. Eur J Radiol 2005;55(1):84-91.

[8] Wang CY, Chung HW, Cho NY, et al. Idiopathic growth hormone deficiency in the morphologically normal pituitary gland is associated with perfusion delay. Radiology 2011;258(1):213-21.

[9] Haque TL, Miki Y, Kashii S, et al. Dynamic MR imaging in Tolosa-Hunt syndrome. Eur J Radiol 2004;51(3):209-17.

[10] Suzuki M, Matsui O, Ueda F, et al. Dynamic MR imaging for diagnosis of lesions adjacent to pituitary gland. Eur J Radiol 2005;53(2):159-67.

[11] Shigematsu Y, Korogi Y, Kitajima M, et al. Abnormal perfusion of the pituitary gland secondary to dural arteriovenous fistulas in the cavernous sinus: dynamic MR findings. AJNR Am J Neuroradiol 2003;24(5):930-6.

[12] Gao R, Isoda H, Tanaka T, et al. Dynamic gadolinium-enhanced MR imaging of pituitary adenomas: usefulness of sequential sagittal and coronal plane images. Eur 
J Radiol 2001;39(3):139-46.

[13] Sato N, Sze G, Endo K. Hypophysitis: endocrinologic and dynamic MR findings. AJNR Am J Neuroradiol 1998;19(3):439-44.

[14] Phatouros CC, Higashida RT, Malek AM, Smith WS, Dowd CF, Halbach VV. Embolization of the meningohypophyseal trunk as a cause of diabetes insipidus. AJNR Am J Neuroradiol 1999;20(6):1115-8.

[15] Maghnie M, Altobelli M, Di Iorgi N, et al. Idiopathic central diabetes insipidus is associated with abnormal blood supply to the posterior pituitary gland caused by vascular impairment of the inferior hypophyseal artery system. J Clin Endocrinol Metab 2004;89(4):1891-6.

[16] Stadnik T, Stevenaert A, Beckers A, Luypaert R, Buisseret T, Osteaux M. Pituitary microadenomas: diagnosis with two-and three-dimensional MR imaging at $1.5 \mathrm{~T}$ before and after injection of gadolinium. Radiology 1990;176(2):419-28.

[17] Ringstad GA, Emblem KE, Holland D, Dale AM, Bjornerud A, Hald JK. Assessment of pituitary adenoma volumetric change using longitudinal MR image registration. Neuroradiology 2012;54(5):435-43.

[18] Breuer FA, Blaimer M, Mueller MF, et al. Controlled aliasing in volumetric parallel imaging (2D CAIPIRINHA). Magn Reson Med 2006;55(3):549-56.

[19] Griswold MA, Jakob PM, Heidemann RM, et al. Generalized autocalibrating partially parallel acquisitions (GRAPPA). Magn Reson Med 2002;47(6):1202-10.

[20] Dietrich O, Raya JG, Reeder SB, Reiser MF, Schoenberg SO. Measurement of signal-to-noise ratios in MR images: influence of multichannel coils, parallel imaging, and reconstruction filters. J Magn Reson Imaging 2007;26(2):375-85.

[21] Goerner FL， Clarke GD. Measuring signal-to-noise ratio in partially parallel imaging MRI. Med Phys 2011;38(9):5049-57.

[22] Saeki N, Tokunaga H, Wagai N, et al. MRI of ectopic posterior pituitary bright spot with large adenomas: appearances and relationship to transient postoperative diabetes insipidus. Neuroradiology 2003;45(10):713-6.

[23] Brooks BS, el Gammal T, Allison JD, Hoffman WH. Frequency and variation of the posterior pituitary bright signal on MR images. AJR Am J Roentgenol 1989;153(5):1033-8.

[24] Saeki N, Hayasaka M, Murai H, et al. Posterior pituitary bright spot in large adenomas: MR assessment of its disappearance or relocation along the stalk. Radiology 2003;226(2):359-65.

[25] Maier C, Riedl M, Clodi M, et al. Dynamic contrast-enhanced MR imaging of the stimulated pituitary gland. Neuroimage 2004;22(1):347-52. 
1

[26] Miki Y, Kanagaki M, Takahashi JA, et al. Evaluation of pituitary macroadenomas with multidetector-row CT (MDCT): comparison with MR imaging. Neuroradiology 2007;49(4):327-33.

[27] Lee HB, Kim ST, Kim HJ, et al. Usefulness of the dynamic gadolinium-enhanced magnetic resonance imaging with simultaneous acquisition of coronal and sagittal planes for detection of pituitary microadenomas. Eur Radiol 2012;22(3):514-8.

[28] Kitamura E, Miki Y, Kawai M, et al. T1 signal intensity and height of the anterior pituitary in neonates: correlation with postnatal time. AJNR Am J Neuroradiol 2008;29(7):1257-60. 


\section{TABLES}

Table 1.

Comparison of air intensity in the sphenoid sinus just below the pituitary lobe between $3 \mathrm{D}$ and $2 \mathrm{D}$.

$2 \mathrm{D}$

\begin{tabular}{ccc}
\hline Pre & $31.58 \pm 8.36(26.46)$ & $79.73 \pm 10.21(12.80)$ \\
1st & $32.44 \pm 8.3(25.58)$ & $82.74 \pm 10.53(12.72)$ \\
2nd & $34.06 \pm 8.62(25.32)$ & $82.41 \pm 11.21(13.6)$ \\
3rd & $34.24 \pm 8.17(23.85)$ & $82.3 \pm 11.24(13.65)$ \\
& & \\
4 th & $35.1 \pm 8.25(23.51)$ & $81.38 \pm 11.23(13.79)$ \\
5th & $34.91 \pm 7.71(22.1)$ & $82.21 \pm 12.05(14.66)$
\end{tabular}

Mean and standard deviation of the air in the sphenoid sinus just below the pituitary lobe are shown. Parenthesis represents coefficient of variance. 
FIGURE CAPTIONS

Fig. 1

Phantom results. SNR image was calculated in a pixel-wise manner from mean signal intensity divided by standard deviation through 30 measurements. G-factor was then calculated from SNR image of 3D VIBE with CAIPIRINHA and 3D VIBE without parallel imaging. G-factor image is shown with MPR coronal (left), sagittal (right upper) and axial (right lower) sections. CAIPIRINHA contributes to G-factor reduction in the central part of the image and slab, whereas G-factor was increased at the peripheral part of the image and slab.

\section{Fig. 2}

A 23-year-old woman. Precontrast (left column), dynamic 1st, 2nd, 3rd, 4th and 5th images from 3D dynamic MR are shown from left to right. Coronal (upper row), axial (middle row) and sagittal (lower row) MPR images are shown. Three-dimensional dynamic MR was performed because of moderate hyperprolactinemia. A dynamically enhanced pituitary stalk is displayed (arrows). A hyperintense posterior lobe was recognized and dynamic images show enhancement of the posterior lobe (arrowheads) and subsequent enhancement of the anterior lobe. 
Fig. 3

A 22-year-old female, the same patient as in Figure 2. Precontrast (left column),

dynamic 1st, 2nd, 3rd, 4th and 5th images from 2D dynamic MR are shown from left to right. Coronal (upper row), axial (middle row) and sagittal (lower row) MPR images are shown. Two-dimensional dynamic MR was performed 1 year before 3D dynamic MR (Fig. 2). Arrows, pituitary stalk; arrowheads, posterior lobe.

\section{Fig. 4}

A 55-year-old man, in a follow-up study for postoperative state of macroadenoma.

Precontrast (left column), dynamic 1st, 2nd, 3rd, 4th and 5th images are shown for 3D

dynamic MR from left to right. Coronal (upper row), axial (middle row) and sagittal (lower row) MPR images are shown. White arrows, posterior lobe; black arrows, stalk. Residual tumor infiltrating the right cavernous sinus is clearly visualized.

\section{Fig. 5}

Values from precontrast (Pre) and dynamic contrast images (1st, 2nd, 3rd, 4th and 5th) are shown for 3D (a) and 2D dynamic images (b) for patients with positive 
1

2

identification of both posterior lobe and stalk (28 patients for 3D, 15 patients for $2 \mathrm{D}$ ).

Error bar represents standard error.

Fig. 6

Enhancement ratio of the posterior lobe and stalk compared by 1st/Pre, 2nd/Pre, 3rd/Pre,

4th/Pre and 5th/Pre between 3D (a) and 2D dynamic images (b). Error bar represents standard error. 
Click here to download high resolution image
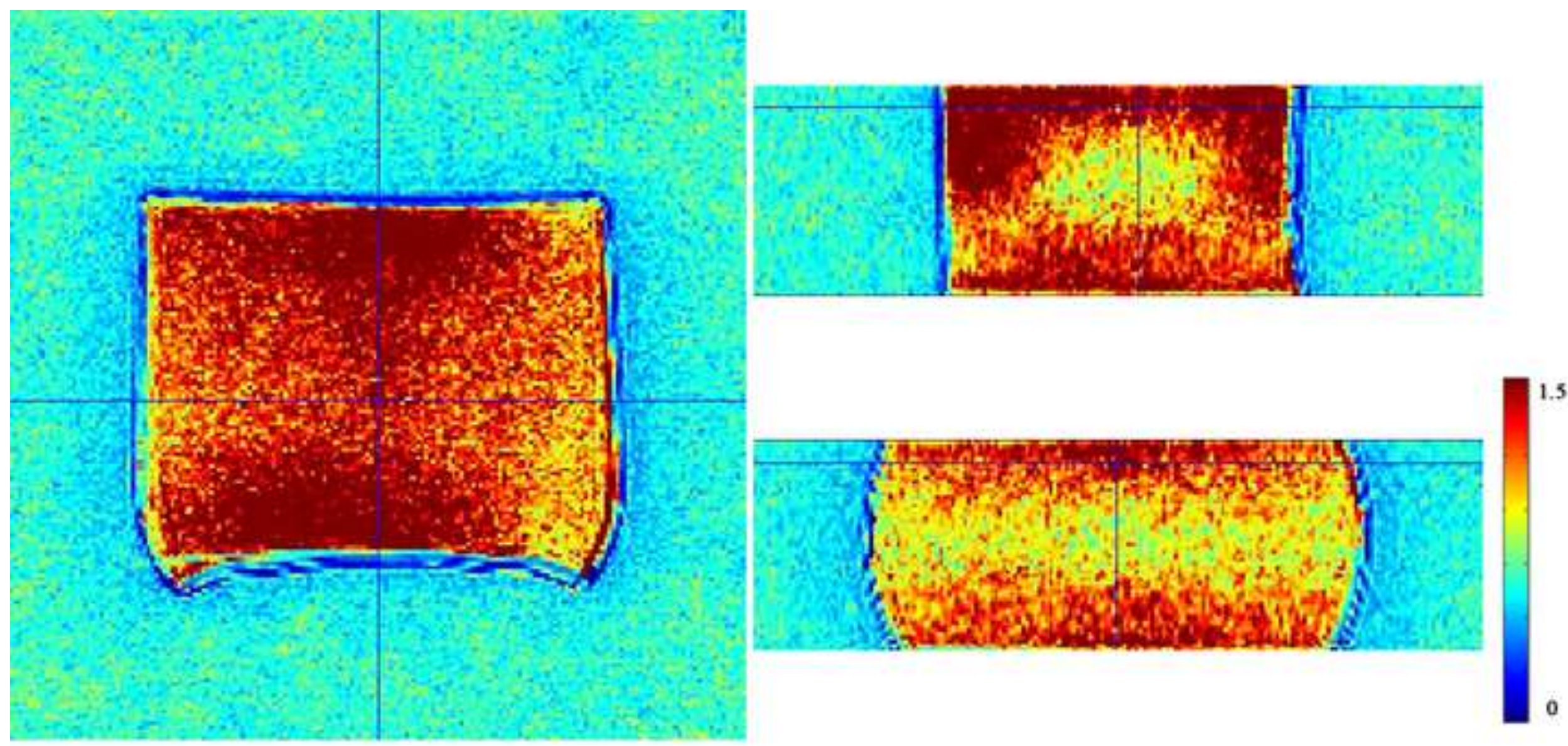

.


Click here to download high resolution image

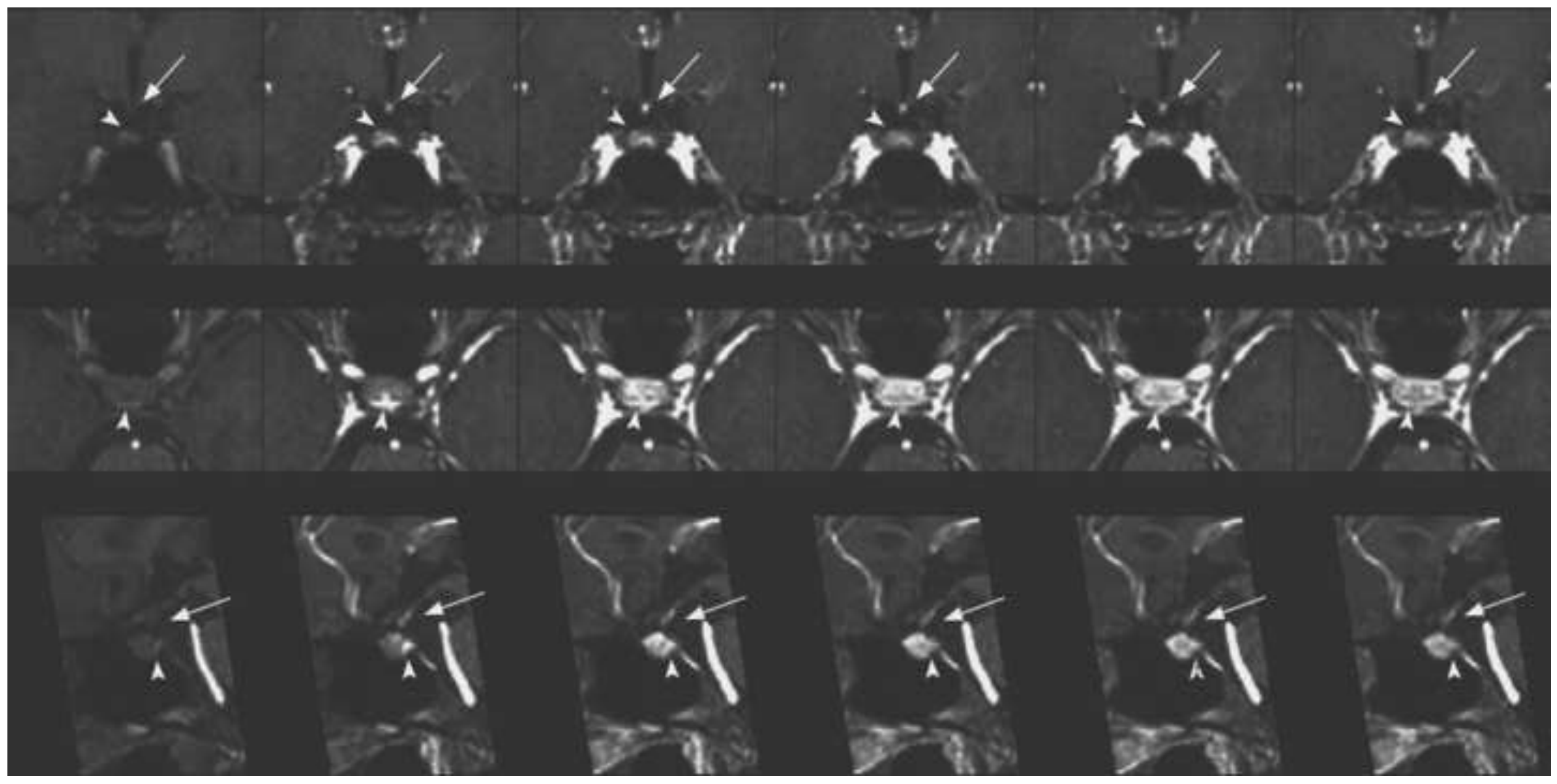

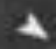

4

is

$\hat{f}^{-1}$

$\hat{7}^{-1}$ 


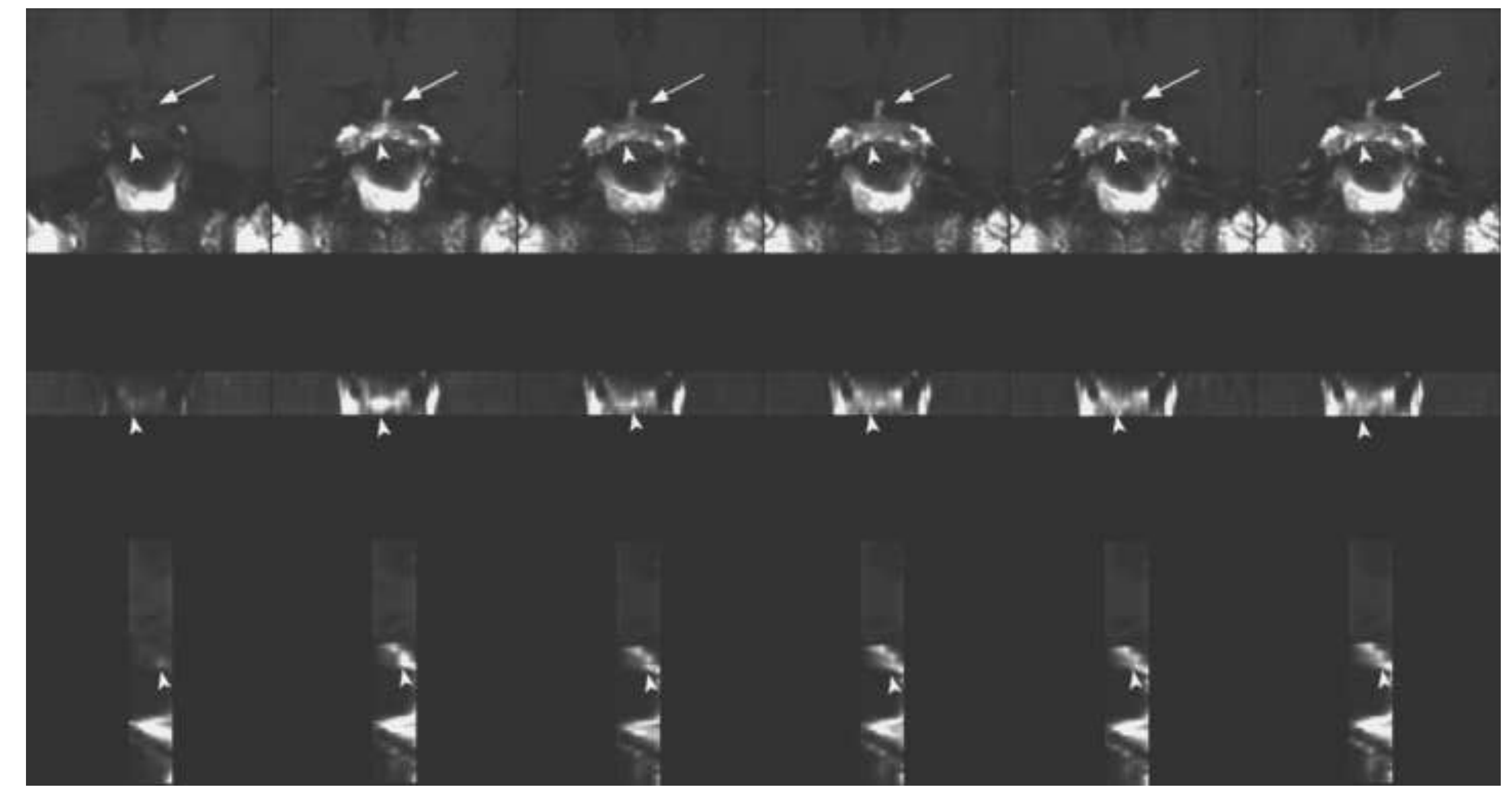

$$
\wedge
$$$$
\text { init }
$$

$1+1$

Ineti

$\mathrm{by}_{\mathrm{i}} \mathrm{il}$

bis 


\section{Figure 4}

Click here to download high resolution image

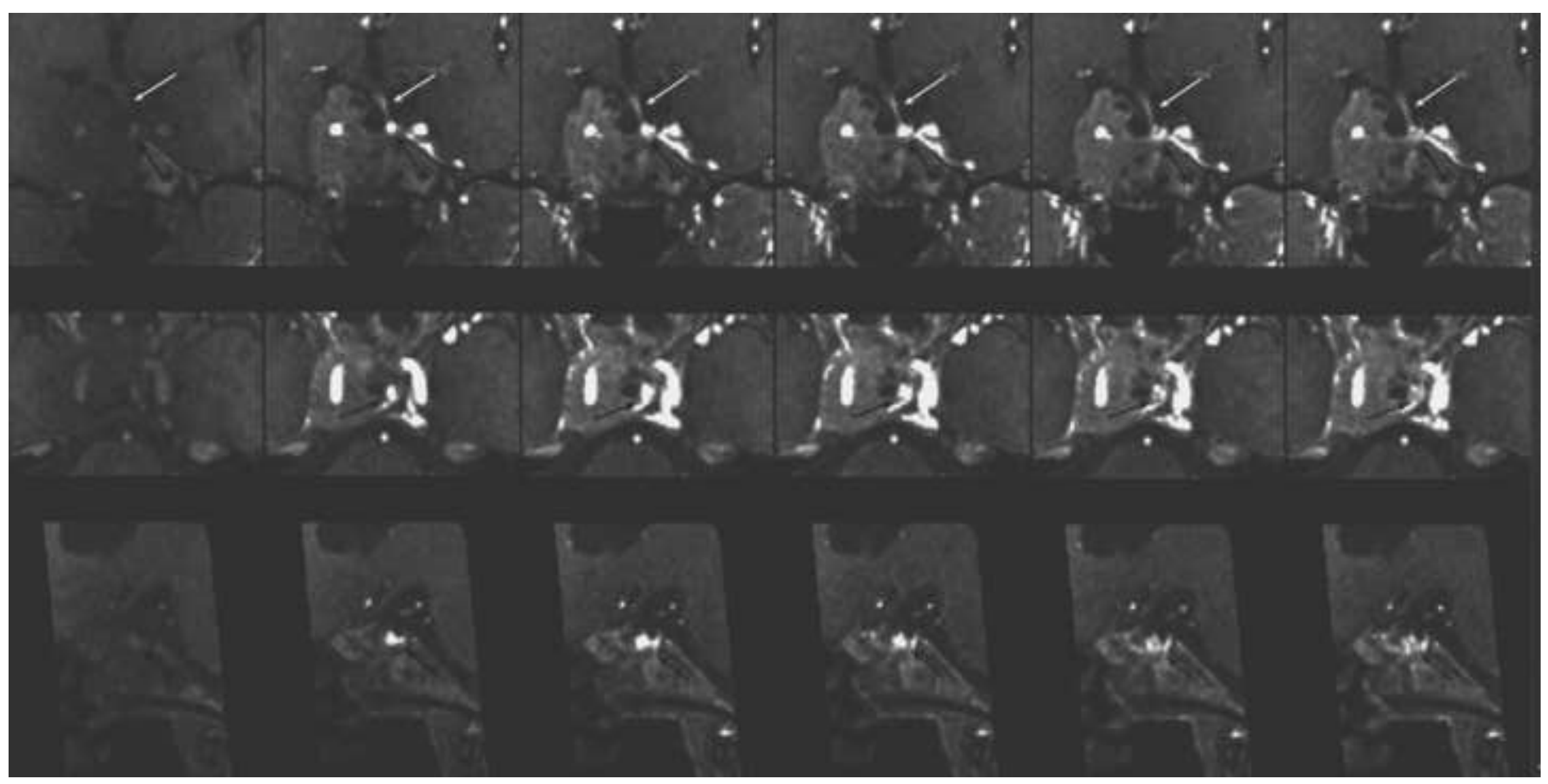


(a) 3D Normalized Value

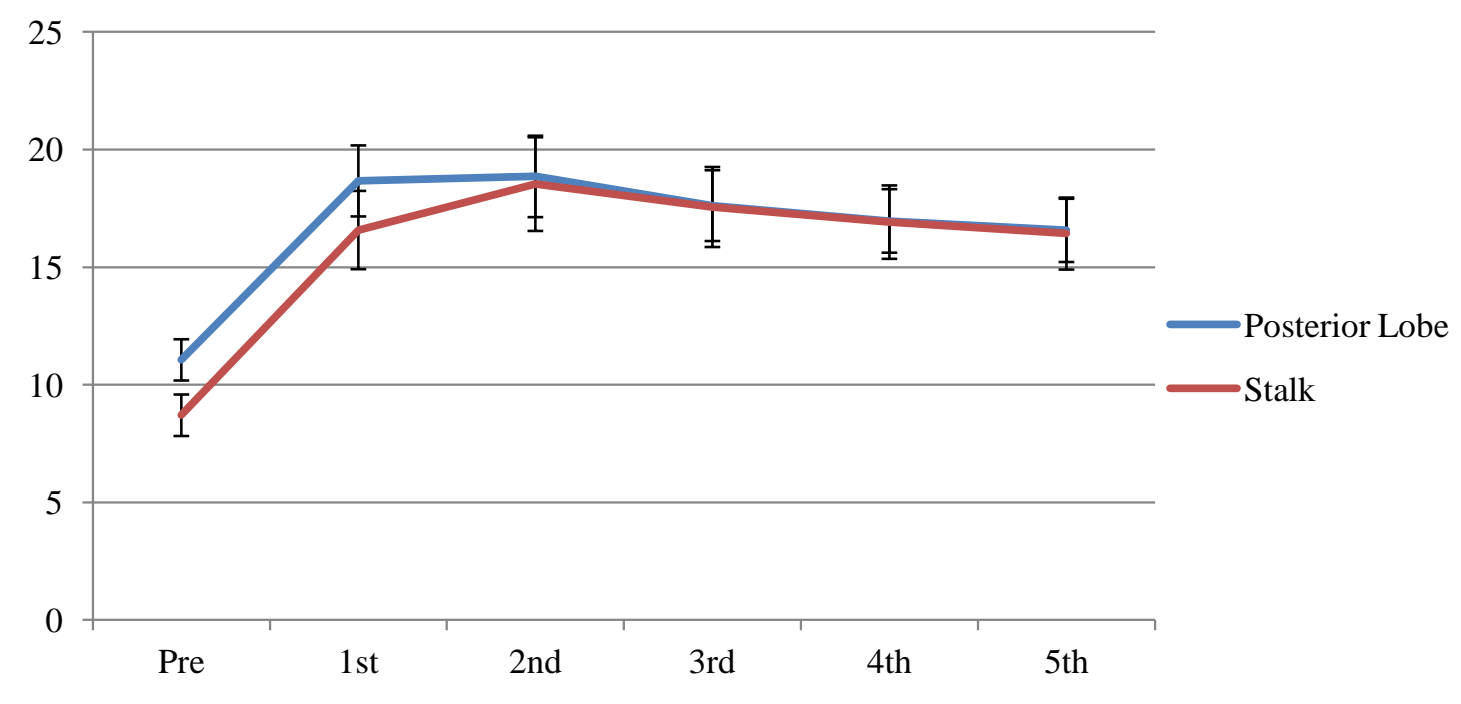

(b) 2D Normalized Value

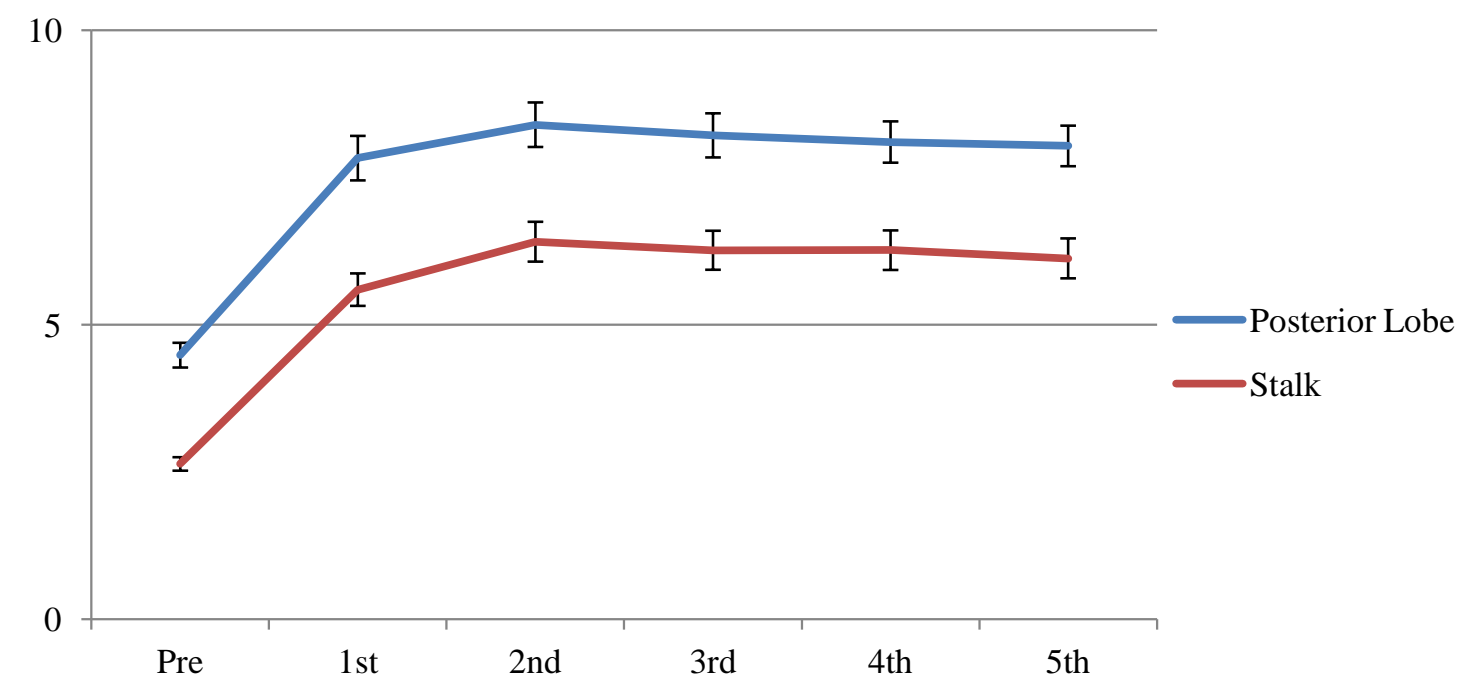

Figure 5

Values from precontrast (Pre) and dynamic contrast images (1st, 2nd, 3rd, 4th and 5th) are shown for 3D (a) and 2D dynamic images (b) for patients with positive identification of both posterior lobe and stalk (28 patients for 3D, 15 patients for 2D). Error bar represents standard error. 
3D Dynamic

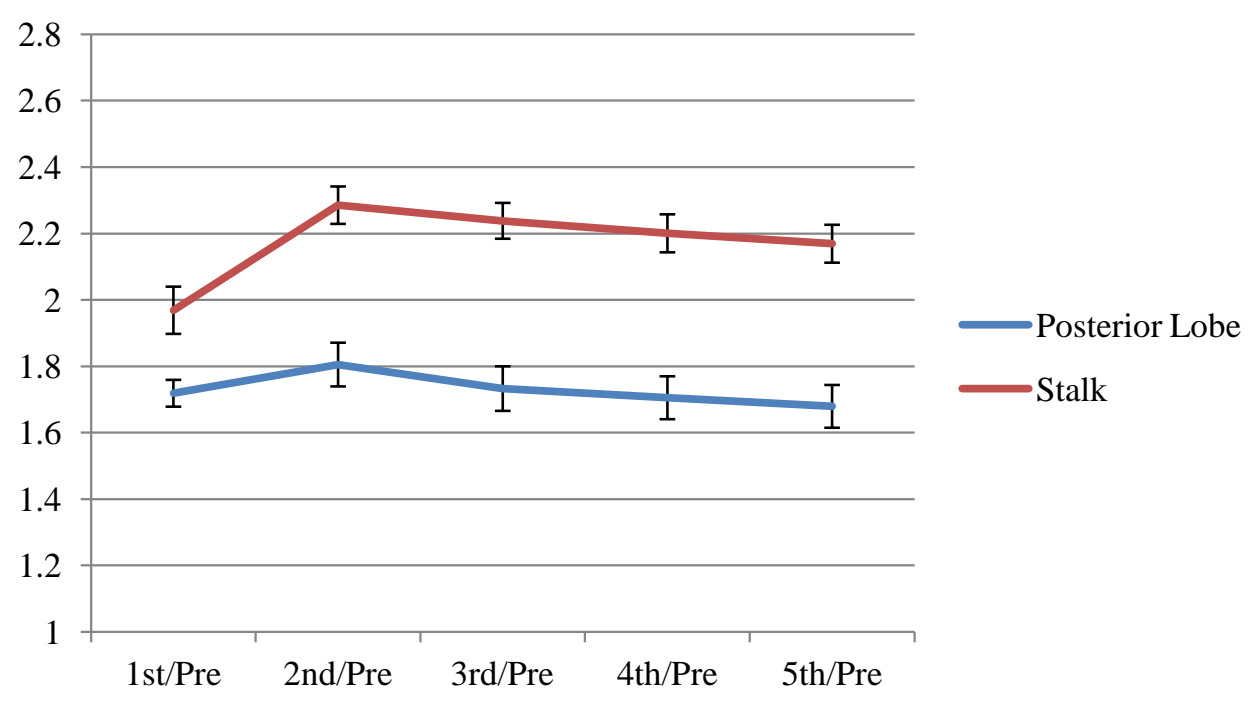

2D Dynamic

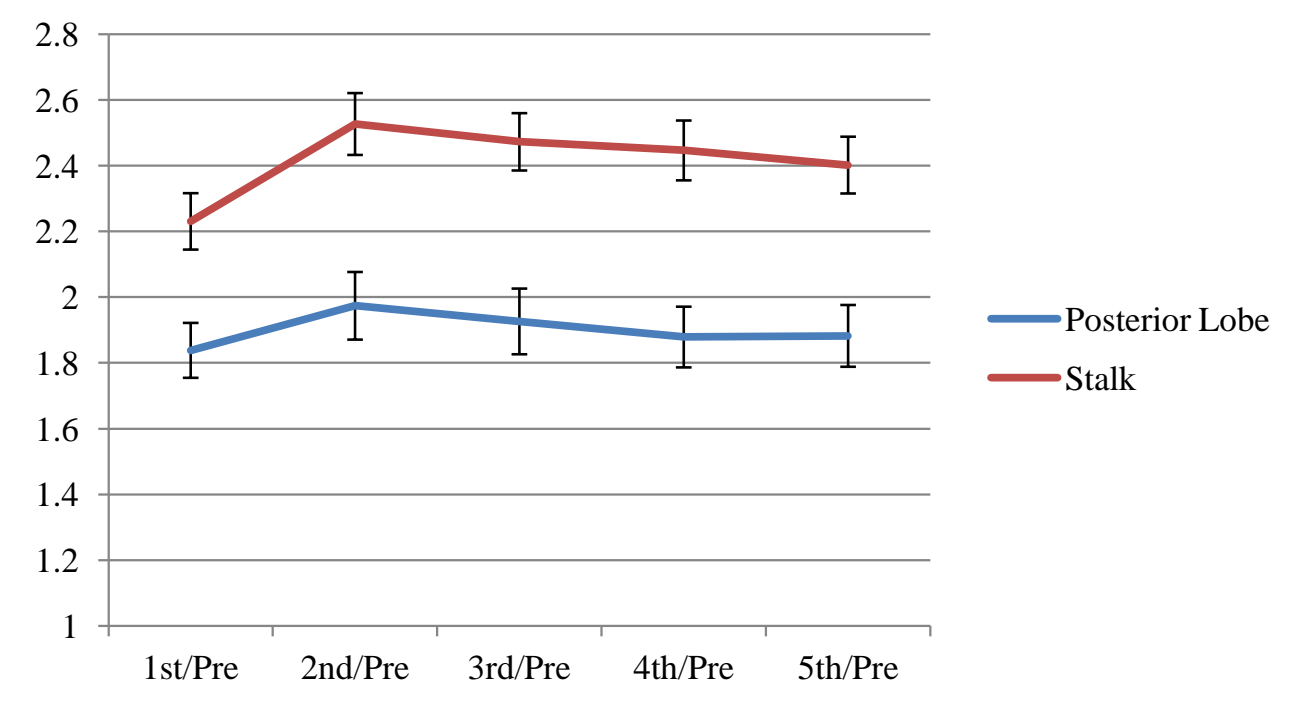

Figure 6

Enhancement ratio of the posterior lobe and stalk compared by 1st/Pre, 2nd/Pre, 3rd/Pre, 4th/Pre and 5th/Pre between 3D (a) and 2D dynamic images (b). Error bar represents standard error. 